\title{
Mycophenolate Mofetil: Use of a Simple Dissolution Technique to Assess Generic Formulation Differences
}

\author{
Emmanuel Scheubel ${ }^{1,2, *}$, Laurent Adamy ${ }^{2}$, and Jean Michel Cardot ${ }^{1}$ \\ 'Biopharmaceutical department, Faculty of Pharmacy, University of Auvergne 28 Place H. Dunant, BP 38, 63001 Clermont-Ferrand, France \\ ${ }^{2}$ F. Hoffmann-La Roche Ltd. Pharmaceutical Division, Galenical \& Analytical Development CH-4070, Basel, Switzerland
}

\begin{abstract}
Mycophenolate mofetil (MMF) is an immunosuppressive agent indicated for the prophylaxis of acute rejection in patients receiving allogeneic renal, cardiac, or hepatic transplants. It is a Biopharmaceutics Classification System Class II substance that has a strongly $\mathrm{pH}$-dependent solubility profile. Consequently, differences in solid-state properties, formulation, and manufacturing processes of MMF can lead to disparities in bioavailability between brands of the same drug. This study was conducted to compare the in vitro dissolution profile of the original MMF innovator brand (CellCept, Roche) with available generic products. Two representative batches of CellCept 500-mg tablets and 14 different generic formulations were tested using different dissolution testing scenarios simulating conditions in the proximal gastrointestinal tract. These scenarios took into account stomach and small intestine media composition, surface tension, $\mathrm{pH}$, increased buffer capacity, and osmolarity after food intake.

Eight of the generic formulations tested passed the quality control dissolution test $(\mathrm{pH} 1.1)$ according to the specification $Q=75 \%$ after 5 min (i.e., all single units $>80 \%$ dissolved), and 12 passed the specification $Q=85 \%$ after 15 min (i.e., all single units $>90 \%$ dissolved). This suggests an almost homogenous dissolution rate between formulations in an acidic environment. However, at $\mathrm{pH} 4.5$, large variations in the in vitro dissolution performance between generic formulations were observed (extremes resulting in greater than $60 \%$ dissolved difference after 30 min). Marked variability was seen among the different generic formulations and the innovator brand, CellCept. In conclusion, important differences exist among the different generic formulations with regard to in vitro performance. As MMF is required for life-long use, changes in drug performance as a result of switching between formulations may have serious clinical consequences (e.g., organ rejection). Therefore, clinical testing is necessary to evaluate the pharmacokinetics and the impact on clinical safety of a change of brands.
\end{abstract}

\section{INTRODUCTION}

A fter the patent protection of an original brand expires, healthcare systems encourage the use of generic medicines. While the economic need to limit healthcare costs by using generics is not questioned, it is important to ensure that patient health is not compromised. Equivalence has to be shown and is usually based on bioequivalence in healthy volunteers (1). However, as generic products are approved based on comparison with only the innovator product (2), one could argue that switching from one generic product to another might give rise to complications due to the potentially greater disparity between two generic products than between any single generic product and the innovator. In particular, a switch from innovator to generic for a lifelong treatment like an immunosuppressive drug may have negative consequences $(3,4)$.

Mycophenolate mofetil (MMF) is an immunosuppressive agent indicated for the prophylaxis of acute rejection in adult recipients of renal, cardiac, or hepatic transplants as well as pediatric recipients of renal transplants. MMF is

* Corresponding author. currently prescribed for lifelong use. MMF is a weak base classified as a BCS Class II substance (5), exhibiting a strong $\mathrm{pH}$-dependent solubility profile (solubility decreases when $\mathrm{pH}$ increases). It is absorbed rapidly and is hydrolyzed by esterases to the active metabolite mycophenolic acid (MPA) (6). The maximum absorption $\left(C_{\max }\right)$ in man is observed after approximately $0.8 \pm 0.36 \mathrm{~h}$ in the fasted state (7).

For the same API, differences in solid-state properties, formulation, excipients, or manufacturing can lead to differences in bioavailability from one finished product to another (8). Because in vivo drug dissolution can be the rate-limiting factor in drug absorption for BCS Class II drugs (9), the use of appropriately designed in vitro dissolution tests can potentially discriminate between formulations with different bioavailability. For MMF, differences in dissolution profiles can potentially be useful predictors of clinical differences (10) since the absorption of this drug with a very short $T_{\max }$ in the fasted state is limited by the dissolution rate.

The aim of this study was to compare the originator brand CellCept 500-mg tablets with generic drug products that were commercially available worldwide using classical in vitro dissolution testing. In addition to the 
Table 1. Dissolution In Vitro Working Conditions Used for Screening of MMF Using Paddle Method at 50 rpm

\begin{tabular}{lcccc}
\hline Medium & pH & Volume $(\mathbf{m L})$ & $\boldsymbol{n}$ & Comments \\
\hline $0.1 \mathrm{~N} \mathrm{HCl}$ & 1.1 & 900 & 12 & QC method, pH of fasted-state stomach \\
\hline Acetate buffer $0.05 \mathrm{M}$ & 4.5 & 900 & 6 & pH of fed-state stomach \\
\hline FaSSIF & 6.5 & 500 & 3 & Fasted-State Simulated Intestinal Fluid \\
\hline FeSSIF & 5.0 & 500 & 3 & Fed-State Simulated Intestinal Fluid \\
\hline FeSSGF & 5.0 & 500 & 3 & Fed-State Simulated Gastric Fluid
\end{tabular}

registered NDA QC method (11), investigations were undertaken to simulate in vitro various conditions that are accounted in vivo.

\section{MATERIAL AND METHODS \\ Material}

Egg lecithin (E PC S, purity $>96 \%$ ) was obtained from Lipoid (Ludwigshafen, Germany), and sodium taurocholate (NaTC, 97\% pure) was used as received from Prodotti Chimici e Alimentari SpA, Basaluzzo, Italy.

Phosphate buffer, sodium chloride (ACS), 37\% hydrochloric acid (fumed), 85\% ortho-phosphoric acid, ethanol (99.9\%), acetic acid, as well as HPLC grade methanol and acetonitrile were purchased from Merck (Darmstadt, Germany). Water was obtained from a Milli-Q water purification system (Millipore, Milford, MA, USA).

Commercial milk (UHT) with at least $3.5 \%$ fat was used.

Two representative marketed batches of Roche CellCept 500-mg tablets and 14 MMF generic samples from various worldwide sources were tested (please note that the generics were purchased in 2008 from countries where the patent had expired.)

\section{Method}

The dissolution profiles were conducted using a Sotax AT 7smart apparatus (Sotax, Allschwill, CH). In addition to the suggested ANDA method for release (paddle $50 \mathrm{rpm}$ in $900 \mathrm{~mL}$ of $0.1 \mathrm{~N} \mathrm{HCl}$ ) (11), different media taking into account stomach and small intestine composition, surface tension, $\mathrm{pH}$, increased buffer capacity, osmolarity, and $\mathrm{pH}$ change after food intake were investigated (12). The different working conditions are presented in Table 1.
Paddle speed was set at $50 \mathrm{rpm}$ and temperature at $37^{\circ} \mathrm{C}$, with $n$ from 3 to 12 units depending on the working conditions or the screening activity. Samples were withdrawn at predefined time intervals from each vessel without replacement. Sampling was automated, and dissolution samples were directly filtered and subsequently measured by a validated UV detection method.

The solubility of the MMF was assessed in each dissolution medium mentioned in Table 1. Duplicate samples were incubated with an excess of compound in a 10-mL volumetric flask at $37^{\circ} \mathrm{C}$ with constant rotation for $4 \mathrm{~h}$, and the dissolved quantity was measured with a validated HPLC-UV detection method.

To simulate the impact of variations in $\mathrm{pH}$ or medium composition on the dissolution behavior of the 500-mg MMF tablets, the comparisons listed in Table 2 were investigated. The dissolution profiles were measured independently in each medium according to Table 1. For this first screening, only two generics, which had shown the highest difference with regard to performance using the NDA method, were compared with CellCept $500 \mathrm{mg}$.

For the comparison of the dissolution profiles, the curves were estimated equivalent in $\mathrm{HCl}$ if they fulfilled the ANDA specification. For CellCept, the limits are $Q=75 \%$ after $5 \mathrm{~min}$ (i.e., Stage 1, all single units greater than $80 \%$ dissolved) and $Q=85 \%$ after 15 min (i.e., Stage 1 , all single units greater than $90 \%$ dissolved). This first step in $\mathrm{HCl}$ will allow selection of formulations to be studied subsequently: a generic fulfilling the requirements and one that does not pass the requirements.

In the other tested media, the similarity factor $f_{2}(13)$ was calculated for each MMF generic versus CellCept, where

\section{Table 2. Overview of the In Vitro Methods Simulating Change After Food Intake}

\begin{tabular}{|c|c|c|c|}
\hline Test & Simulated Food Intake & Compared Media and pH & Comments \\
\hline \multirow[t]{2}{*}{1} & Stomach & $\mathrm{HCl} \mathrm{pH} 1.2$ & $\begin{array}{c}\text { Gastric pH decreases continuously after meal ingestion from } \mathrm{pH} 6.4 \text { to } 2.7 \text {. } \\
\text { Middle condition was chosen (12). }\end{array}$ \\
\hline & & FeSSGF pH 5.0 & \\
\hline \multirow[t]{2}{*}{2} & Small intestine & FaSSIF pH 6.5 & \multirow{2}{*}{$\begin{array}{l}\text { To simulate pre- and postprandial bile secretion, in upper small intestine before } \\
\text { and after food intake }\end{array}$} \\
\hline & & FeSSIF pH 5.0 & \\
\hline \multirow[t]{2}{*}{3} & $\mathrm{pH}$ variation in stomach & $\mathrm{HCl} \mathrm{pH} 1.1$ & \multirow{2}{*}{$\begin{array}{l}\text { pH } 4.5 \text { was chosen as non-sink method, allowing } 1 \text { tablet }(500 \mathrm{mg}) \text { to dissolve in } \\
\qquad 900 \mathrm{~mL}\end{array}$} \\
\hline & & Acetate $\mathrm{pH} 4.5$ & \\
\hline
\end{tabular}


Table 3. Solubility of MMF in Different Media According to In Vitro Working Conditions

\begin{tabular}{lc}
\hline Medium & Solubility of MMF \\
\hline FeSSGF pH 5.0 & $290 \mathrm{mg} / 500 \mathrm{~mL}$ \\
\hline FaSSIF pH 6.5 & $38 \mathrm{mg} / 500 \mathrm{~mL}$ \\
\hline Blank FaSSIF & $35 \mathrm{mg} / 500 \mathrm{~mL}$ \\
\hline FeSSIF pH 5.0 & $548 \mathrm{mg} / 500 \mathrm{~mL}$ \\
\hline Blank FeSSIF & $324 \mathrm{mg} / 500 \mathrm{~mL}$ \\
\hline $0.1 \mathrm{~N} \mathrm{HCl} \mathrm{pH} 1.1$ & $4270 \mathrm{mg} / \mathrm{L}$ \\
\hline Acetate pH 4.5 & $600 \mathrm{mg} / \mathrm{L}$ \\
\hline
\end{tabular}

applicable (at least three points in the ascending part of the curve with the maximum one above $85 \%$ ). The FDA (14) and EMEA (15) recommend that two dissolution profiles are similar if $f_{2}$ is between 50 and 100 .

\section{RESULTS}

\section{Solubility}

Table 3 summarizes the results of the solubility study; sink conditions were reached only in $0.1 \mathrm{~N} \mathrm{HCl}$.

\section{Dissolution}

The dissolution profiles of the various formulations in $0.1 \mathrm{~N} \mathrm{HCl}$ are presented in Figure 1. Table 4 presents the main results according to ANDA specifications.

Four generics out of 14 did not pass the S1 ANDA requirements (28\%), and two out of 14 did not correspond to the ANDA method after 15 min (14\%). Generic 2, which was closer to the reference formulation, and generic 4, which dramatically failed, were selected for further investigation to select an alternative medium to $0.1 \mathrm{~N} \mathrm{HCl}$.

Table 5 summarizes the finding observed in various media. A similar rank-order was observed in all media for the three formulations; generic 2 was always equivalent to

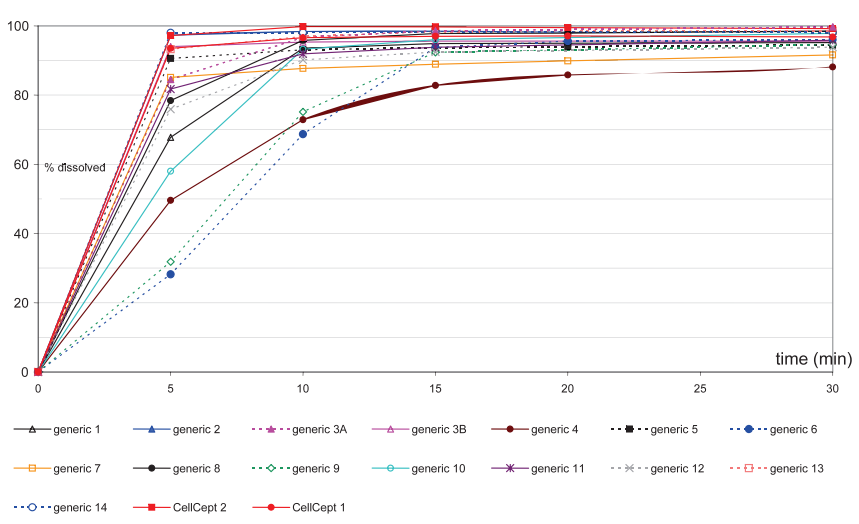

Figure 1. Dissolution profiles according to NDA method. Results for Roche CellCept are in red $(n=12)$. After $5 \mathrm{~min}, 2 \%<S D<8 \%$, and after $15 \mathrm{~min}$ $S D<2 \%$. The observed variations within the tested tablets batches are very low.

Dissolution Technologies | FEBRUARY 2012 the reference and generic 4 was always lower than the reference. Figure 2 and Table 6 summarize the differences observed in acetate buffer (the simplest medium that emphasizes differences). The dissolution under not strictly sink conditions could be questionable but highlights differences that could have a clinical relevance.

\section{Discussion}

MMF exhibited a typical pH-dependent solubility profile. Sink conditions (three times saturation in dissolution volume) were fulfilled only in $0.1 \mathrm{~N} \mathrm{HCl}$. In 900 $\mathrm{mL}$ of $\mathrm{pH} 4.5$ acetate buffer, the entire tablet dose could be dissolved. Comparison of the solubility of the blank FaSSIF and blank FeSSIF versus FaSSIF and FeSSIF, respectively, suggests that the impact of bile salt and lecithin were well pronounced and consequently food intake can impact the solubility of MMF in vivo.

According to the ANDA method (Figure 1), different profiles were observed for the generics in comparison with the reference CellCept 500 mg. Nine of the 14 tested generics successfully passed the ANDA dissolution test (Table 1) according to the current specification $Q=75 \%$ after $5 \mathrm{~min}$, and 12 successfully passed according to the specification $Q=85 \%$ after $15 \mathrm{~min}$. Four of them only fulfilled the acceptance criteria after USP Stage 2, and two out of the 14 failed our current specifications. These results indicate an almost homogenous dissolution rate in an acidic environment even if at least two formulations do not comply with the dissolution specifications of FDA, which suggests that they could not be marketed in the United States. Due to the limited amount of available tablets for each generic, two generics ( 2 and 4 ) representing the highest and the lowest value found after 15 min with the NDA method, were selected for dissolution method screening. The impact of food intake and $\mathrm{pH}$ change on MMF dissolution performance was evaluated by comparing the obtained profiles measured in different media independently as mentioned in Table 2.

The simulation of $\mathrm{pH}$ change and the impact of medium composition in the stomach as observed in Figure 3 (Test 1) suggest a similar impact of dissolution media for both CellCept and the tested generics (i.e., after $15 \mathrm{~min}$ decrease of approx $60 \%$ from $\mathrm{HCl}$ to FeSSGF) respecting the ranking observed in $0.1 \mathrm{~N} \mathrm{HCl}$. The similarity factor $f_{2}$ confirmed that generic 4 is not comparable to CellCept, whereas generic 2 resulted in a similar profile (Table 5).

The impact of $\mathrm{pH}$ change and medium composition in the upper small intestine before and after food intake to simulate pre- and postprandial bile secretion, as shown in Figure 4 (Test 2), suggests a similar behavior for CellCept and generic 2 (i.e., after $15 \mathrm{~min}$ decrease of approx. 50\% from FeSSIF to FaSSIF). Generic 4 showed a significant lower profile in FeSSIF and consequently a less pronounced decrease in FaSSIF. In fact, at pH 6.5 (FaSSIF), the maximum solubility was reached ( $37.5 \mathrm{mg}$ in $500 \mathrm{~mL}$ ), which resulted in a plateau at approximately $7-8 \%$ for all 
Table 4. Dissolution Results According to NDA Method

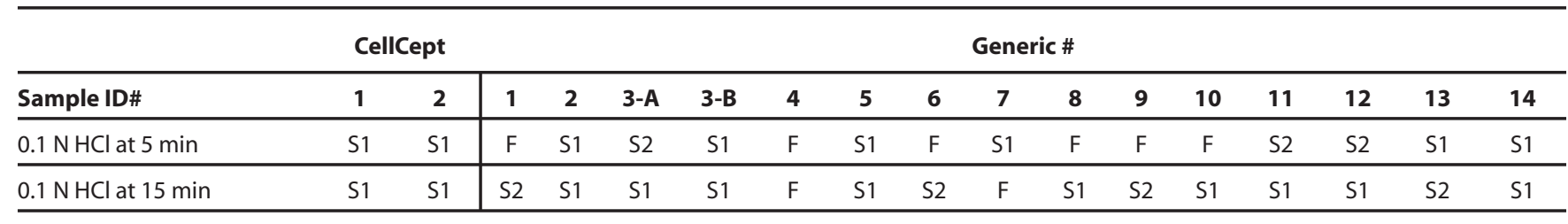

S1 and S2 correspond to the USP stage of acceptance according to pharmacopeia (e.g., USP <711>).

F: fails acceptance criteria.

tested samples. At pH 5.0 (FeSSIF) conditions in which the complete dose can theoretically be solubilized (500 $\mathrm{mg}$ in $500 \mathrm{~mL}$ ), significant differences in performance for generics 2 and 4 were observed the $f_{2}$ factor for generic 4 was much less than 50 .

Simulation of $\mathrm{pH}$ change was further investigated by using common dissolution media as shown in Figure 5 (Test 3). The dissolution profile of generic 2 showed a decrease of about $30 \%$, which was slightly greater than CellCept, whereas generic 4 resulted in $20 \%$ dissolved only after $15 \mathrm{~min}$. The lowest observed $f_{2}$ factor was for generic 4 (Table 5).

Based on this screening, the highest discriminatory power is observed with pH 5.0 FeSSIF and pH 4.5 acetate buffer. At these $\mathrm{pH}$ values and with the working conditions used, comparable solubility exists, and similar profiles can be observed (see Figure 6). In practice, the acetate buffer is advantageous because it is simple to prepare and, in comparison to more complex approaches using simulated intestinal fluids (16-18), it has the potential to serve as both a robust dissolution method and a biorelevant method with a high discriminative power. This method was therefore set up for further investigation of the available generics.

Large variations of in vitro dissolution performance from generic to generic were observed (Figure 2 and Table 6). The $\mathrm{pH} 4.5$ acetate buffer method clearly identified two levels of performance in vitro from 15 min onwards. Extremes resulted in less than $50 \%$ dissolved after $30 \mathrm{~min}$ for 4 generics and less than $30 \%$ dissolved for one generic out of the 14 tested variants. Significant interbatch variability (generics $3 \mathrm{~A}$ and $3 \mathrm{~B}$ ) was also found among generics in comparison with CellCept tablets $500 \mathrm{mg}$

Table 5. Comparison of Dissolution Profiles Using Similarity Factor $f_{2}$ in Various Media

\begin{tabular}{lccc}
\hline Medium & CellCept 2 & Generic 2 & Generic 4 \\
\hline FaSSIF & $*$ & $*$ & $*$ \\
\hline FeSSIF & ref & 71 & 19 \\
\hline FeSSGF & ref & 60 & 29 \\
\hline $\mathrm{HCl}$ & ref & $* *$ & 26 \\
\hline Acetate & ref & 71 & 15 \\
\hline
\end{tabular}

* All profiles lie under $10 \%$ release and are superimposable.

**Not applicable, but profiles are superimposable.
(CellCept 1 and 2 resulted in very similar profiles with $f_{2}=82$ ).

The natural $\mathrm{pH}$ in the fasted state of the stomach is 4.5 (19), and a direct consequence of the marked difference in performance at this $\mathrm{pH}$ could be a strong decrease in the release rate for some generics, whereas CellCept tablets and other generics remained fast dissolving (> 80\% after $30 \mathrm{~min}$ ). Under fasted conditions, the release of MMF in patients having high variation of gastric $\mathrm{pH}$ or achloridia could be directly impacted depending on the generics quality. High performance variations in the fed-state stomach (with $\mathrm{pH}$ from 6 to 3 ) can then be anticipated as well (11). In addition, with a drug having a $T_{\max }$ of approximately $30 \mathrm{~min}$, the difference observed in Figure 2 for some generics would result in a significant delay of the absorption kinetics $\left(T_{\max }\right.$ and $C_{\max }$ ) at higher $\mathrm{pH}$ levels since the drug is potentially not completely dissolved at gastric emptying (after 15-30 min in fasted conditions). It can be clearly shown that different qualities of generics exist and that variable performance can occur from generic to generic even if the therapeutic impact could not be anticipated from those results. Special attention should be given to the generics with the lower results. As generic drugs are approved based only on a comparison with the innovator brand, switching between generic products may possibly lead to greater disparity than switching between a generic product and the innovator brand-with unknown clinical consequences. In view of these uncertainties, further research should be done to evaluate

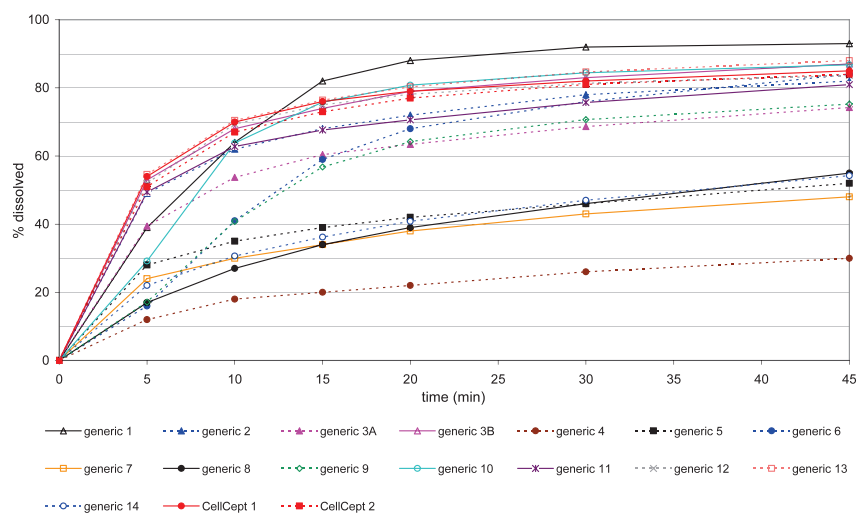

Figure 2. Dissolution profiles of all tested generics at $\mathrm{pH}$ 4.5. Roche CellCept results are in red. After $5 \mathrm{~min}, \mathrm{SD}<5 \%$, after $15 \mathrm{~min}<2 \%$, and after $45 \mathrm{~min}$ $<2 \%$. 
Table 6. Dissolution Results According to Acetate Method

\begin{tabular}{lcc|ccccccccccccccc}
\hline \multicolumn{11}{c}{ CellCept } & \multicolumn{110}{c}{ Generic \# } \\
\hline Sample ID & 1 & 2 & 1 & 2 & $3-\mathrm{A}$ & $3-\mathrm{B}$ & 4 & 5 & 6 & 7 & 8 & 9 & 10 & 11 & 12 & 13 & 14 \\
\hline $\boldsymbol{f}_{\mathbf{2}}$ in acetate & 81 & ref & 52 & 71 & 47 & 83 & 15 & 26 & 37 & 23 & 24 & 36 & 53 & 67 & 89 & 73 & 25 \\
\hline Verdict & $\mathrm{P}$ & $\mathrm{P}$ & $\mathrm{P}$ & $\mathrm{P}$ & $\mathrm{F}$ & $\mathrm{P}$ & $\mathrm{F}$ & $\mathrm{F}$ & $\mathrm{F}$ & $\mathrm{F}$ & $\mathrm{F}$ & $\mathrm{F}$ & $\mathrm{P}$ & $\mathrm{P}$ & $\mathrm{P}$ & $\mathrm{P}$ & $\mathrm{F}$ \\
\hline
\end{tabular}

Pass (P) and fail (F) the $f_{2}$ acceptance criteria.

the pharmacokinetic and safety profiles of generics with lower dissolution results and to determine if they are all therapeutically equivalent. For MMF, change in performance can dramatically affect the risk associated with the medication (e.g., acute rejection in patients).

The FDA draft guidance Average, Population, and Individual Approaches to Establishing Bioequivalence (20), which was never adopted, defined two interesting concepts, switchability and prescribability, that could be of interest for typical drugs like antirejection drugs.

Prescribability refers to the clinical setting in which a practitioner prescribes a drug product to a patient for the first time. In this setting, the prescriber relies on an understanding that the average performance of the drug product has been well characterized and relates in some definable way to the safety and efficacy information from clinical trials.

Switchability refers to the setting in which a practitioner transfers a patient from one drug product to another. This situation arises with generic substitution, as well as with certain postapproval changes by an innovator or generic firm in the formulation, manufacture, or both of a drug product. Under these circumstances, the prescriber and patient should be assured that the newly administered drug product will yield safety and efficacy comparable to that of the product for which it is being substituted.

In this case, MMF could be considered as a prescribable drug. These investigations highlight the importance of

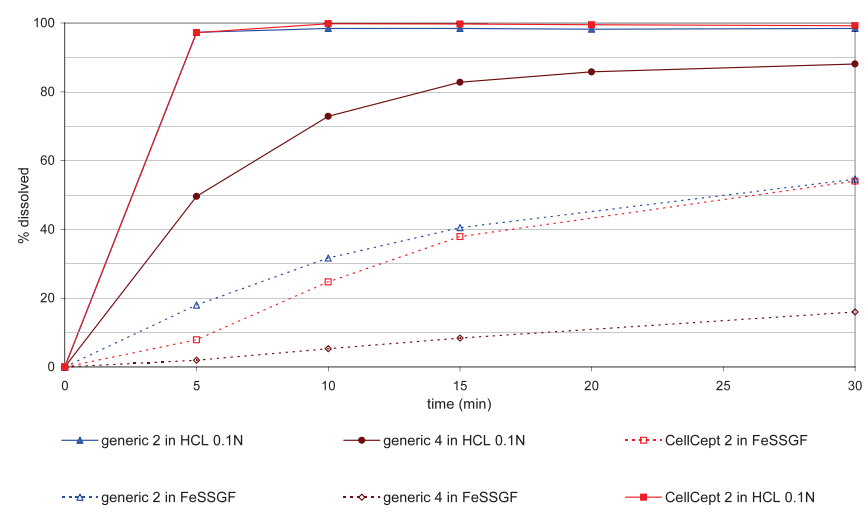

Figure 3. Results for Test 1-Dissolution profiles of CellCept (red) and two generics in media simulating fasted state $(0.1 \mathrm{~N} \mathrm{HCl})$ and fed state (FeSSGF pH 5.0, dotted line) in the stomach. The standard deviation after 15 min lies at maximum $2 \%$. The observed variations within the tested tablets batches are very low. developing discriminating dissolution methods that take into account the physicochemical properties of the drug as well as the characteristics of the formulation. The methods do not necessarily require a complex setting; they need to match the key parameter likely to impact the performance. The results reported here were generated with a limited number of tablets from only one or two lots of each generic or manufacturer. It is unknown whether these results are representative of other lots. Nevertheless, huge differences were observed and some of them were even out of specification using the current method proposed by NDA. The acetate buffer medium highlights the differences and is able to discriminate easily between batches that fully comply in $\mathrm{HCl}$. The $\mathrm{pH} 4.5$ acetate method discriminated eight variants out of 14 , whereas the $\mathrm{HCl}$ method identified two variants that failed the acceptance criteria after $15 \mathrm{~min}$. Considering the nature of the drug, a bioequivalence study is mandatory according to the FDA guidance (21), and studies in fasted and fed states are recommended (22) because a therapeutic failure could not be allowed for this class of drug. A simple dissolution using a standard acetate buffer medium will address potential differences and could help to ensure greater similarity between formulations. Such a simple method, due to its discriminative power, could help generic development before any in vivo studies and, as a quality control tool, ensure better homogeneity between batches, which is important to the efficacy and safety of the drug.

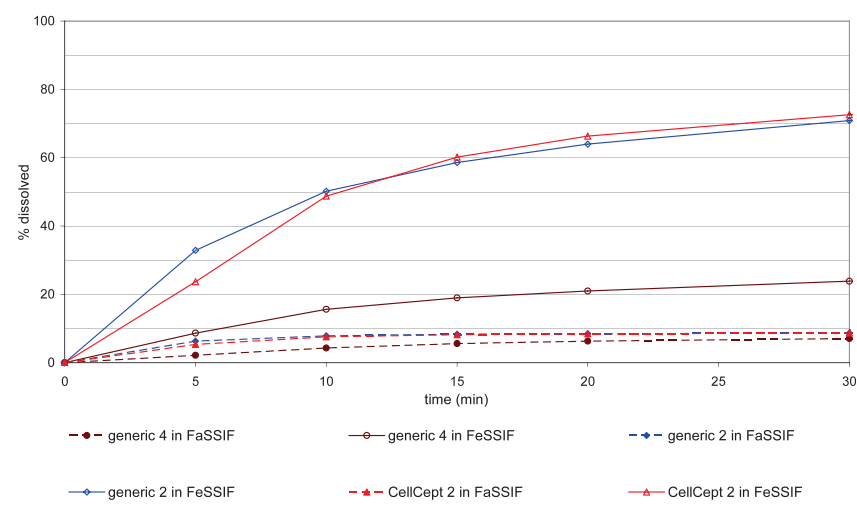

Figure 4. Results for Test 2-Dissolution profiles of CellCept (red) and two generics in media simulating fasted state (FaSSIF, dotted line) and fed state (FeSSIF) in the small intestine.The standard deviation after 15 min lies at maximum $2 \%$. The observed variations within the tested tablets batches are verylow. 


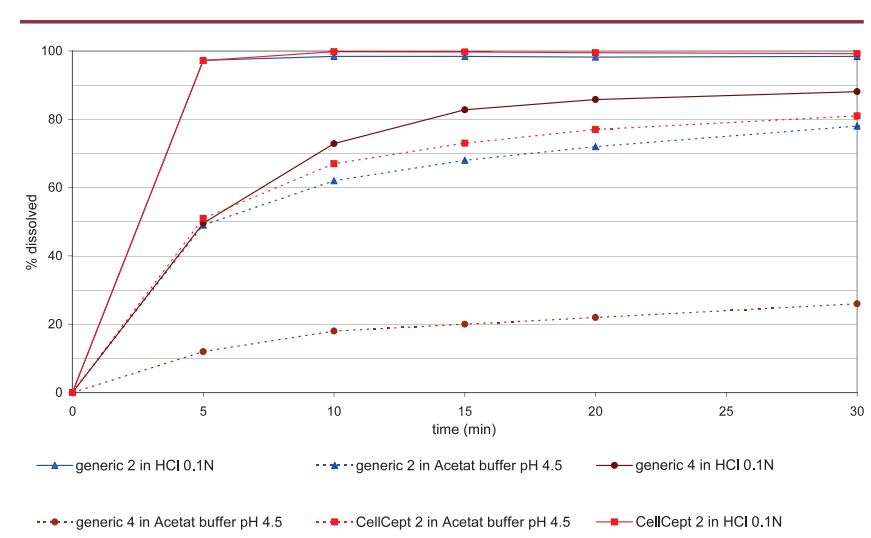

Figure 5. Results for Test 3-Dissolution profiles of CellCept (red) and two generics in media simulating $\mathrm{pH}$ variation in the stomach, $0.1 \mathrm{~N} \mathrm{HCl}$ and $\mathrm{pH}$ 4.5 acetate (dotted line).

\section{CONCLUSION}

In summary, the generics and CellCept tablets $(500 \mathrm{mg}$ ) dissolution profiles obtained with an alternate dissolution method at $\mathrm{pH} 4.5$ suggest that important differences may exist among the different generics with regard to in vitro performance. For MMF, differences in dissolution profiles can be useful predictors of clinical differences, since the absorption of this drug with a very short $T_{\max }$ in the fasted state is potentially limited by the dissolution rate. Taking into account that MMF is currently prescribed for lifelong use, additional clinical testing may be necessary to evaluate the pharmacokinetics and clinical safety impact of a switch from one generic product to another.

If these in vitro findings are confirmed by clinical studies and significant implications for safety and effectiveness are proven, they should be considered by clinicians to potentially safeguard patients who choose to purchase generic drugs. In this case, MMF formulations should behave similarly in $\mathrm{HCl}$ and $\mathrm{pH} 4.5$ acetate buffer before being developed further by new formulators. This prior in vitro knowledge, even if it could be over discriminative, should be leveraged as a resource to aid in the development,

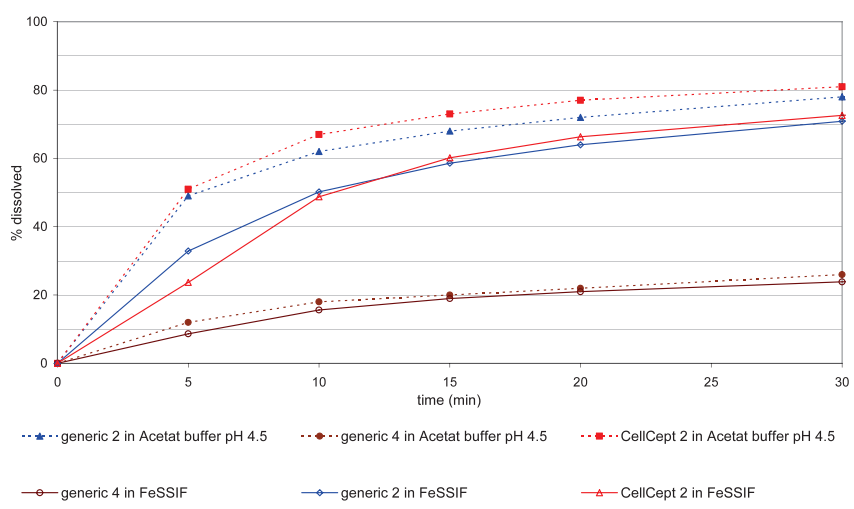

Figure 6. Dissolution profiles of CellCept (red) and two generics in pH 4.5 acetate (dotted line) and pH 5.0 FeSSIF. justify tests and specifications for new products, and ensure safety and similarity of generics for the patients.

\section{ACKNOWLEDGMENTS}

The authors acknowledge C. Keiflin, P.Wininger, C. Maureta, M. Brach, and Y. Ducommun for technical assistance and B. Fischer for managerial support.

\section{REFERENCES}

1. Masri, M. A.; Rizk, S.; Attia, M. L.; Barbouch, H.; Rost, M. Bioavailability of a new generic formulation of mycophenolate mofetil MMF 500 versus CellCept in healthy adult volunteers. Transplant. Proc. 2007, 39 (4), 1233-1236.

2. Bioequivalence Recommendations for Specific Products; Guidance for Industry; U.S. Department of Health and Human Services, Food and Drug Administration, Center for Drug Evaluation and Research (CDER), U.S. Government Printing Office:Washington, DC, 2010.

3. Sabatini, S.; Ferguson, R. M.; Helderman, J.H.; Hull, A. R.; Kirkpatrick, B. S.; Barr, W. H. Drug substitution in transplantation: a National Kidney Foundation White Paper. Am. J. Kidney Dis. 1999, 33 (2), 389-397.

4. Henderson, J. D.; Esham, R. H. Generic substitution: issues for problematic drugs. South. Med.J. 2001, 94 (1), 16-21.

5. Yu, L. X.; Amidon, G. L.; Polli, J. E.; Zhao, H.; Mehta, M. U.; Conner, D. P.; Shah, V. P.; Lesko, L. J.; Chen, M. L.; Lee, V.H.; Hussain, A. S. Biopharmaceutics classification system: the scientific basis for biowaiver extensions. Pharm. Res. 2002, 19 (7), 921-925.

6. Placebo-controlled study of mycophenolate mofetil combined with cyclosporin and corticosteroids for prevention of acute rejection. European Mycophenolate Mofetil Cooperative Study Group. Lancet 1995, 345 (8961), 1321-1325.

7. CellCept Full Prescribing Information, 2010. Genentech CellCept Web site. http://www.gene.com/ gene/products/information/cellcept/pdf/pi.pdf (accessed Jan 11, 2012).

8. Videau, J.-Y.Making medicines safe. Bull. World Health Organ. 2001, 79 (2), 87.

9. Emami, J. In vitro-in vivo correlation: from theory to applications. J. Pharm. Pharm. Sci. 2006, 9 (2), 169-189.

10. Benet, L. Z.; Goyan, J. E. Bioequivalence and narrow therapeutic index drugs. Pharmacotherapy 1995, 15 (4), 433-440.

11. Dissolution Methods Database. U.S. Food and Drug Administration Web site. http://www.accessdata.fda. gov/scripts/cder/dissolution/dsp_SearchResults_ Dissolutions.cfm?PrintAll=1 (accessed Jan 12, 2012).

12. Jantratid, E.; Dressman, J. Biorelevant Dissolution Media Simulating the Proximal Human Gastrointestinal Tract: An Update. Dissolution Technol. 2009, 16 (3), 21-25.

13. Moore, J.W.; Flanner, H. H. Mathematical Comparison of Dissolution Profiles. Pharm. Tech. 1996, 20, 64-74.

Dissolution Technologies | FEBRUARY 2012 
14. Immediate Release Solid Oral Dosage Forms, Scale-Up and Postapproval Changes: Chemistry, Manufacturing, and Controls, In Vitro Dissolution Testing, and In Vivo Bioequivalence Documentation; Guidance for Industry; U.S. Department of Health and Human Services, Food and Drug Administration, Center for Drug Evaluation and Research (CDER), U.S. Government Printing Office: Washington, DC, 1995.

15. Note For Guidance on Quality of Modified Release Products: A. Oral Dosage Forms, B. Transdermal Dosage Forms; Section I (Quality); CPMP/QWP/604/96; Committee for Proprietary Medicinal Products (CPMP), European Medicines Agency: London, 1999.

16. Lue, B. M.; Nielsen, F. S.; Magnussen, T.; Schou, H. M.; Kristensen, K.; Jacobsen, L. O.; Müllertz, A. Using biorelevant dissolution to obtain IVIVC of solid dosage forms containing a poorly-soluble model compound. Eur. J. Pharm. Biopharm. 2008, 69 (2), 648-657.

17. Dressman, J. B.; Amidon, G. L.; Reppas, C.; Shah, V.P. Dissolution testing as a prognostic tool for oral drug absorption: immediate release dosage forms. Pharm. Res. 1998, 15 (1), 11-22.

18. Galia, E.; Nicolaides, E.; Hörter, D.; Löbenberg, R.; Reppas, C.; Dressman, J. B. Evaluation of various dissolution media for predicting in vivo performance of Class I and II drugs. Pharm. Res. 1998, 15 (5), 698-705.
19. Kararli,T.T.Comparison of the gastrointestinal anatomy, physiology, and biochemistry of humans and commonly used laboratory animals. Biopharm. Drug Dispos. 1995, 16 (5), 351-380.

20. Average, Population, and Individual Approaches to Establishing Bioequivalence, Draft Guidance; Guidance for Industry; U.S. Department of Health and Human Services, Food and Drug Administration, Center for Drug Evaluation and Research (CDER), U.S. Government Printing Office:Washington, DC, 1999. http://www.fda.gov/OHRMS/DOCKETS/98fr/3657gd1. pdf (accessed Jan 12, 2012).

21. Bioavailability and Bioequivalence Studies for Orally Administered Drug Products_-General Considerations; Guidance for Industry; U.S. Department of Health and Human Services, Food and Drug Administration, Center for Drug Evaluation and Research (CDER), U.S. Government Printing Office:Washington, DC, 2003.

22. Draft Guidance on Mycophenolate Mofetil Hydrochloride; U.S. Department of Health and Human Services, Food and Drug Administration, Center for Drug Evaluation and Research (CDER), U.S. Government Printing Office:Washington, DC, 2007. http://www.fda.gov/downloads/Drugs/GuidanceCom plianceRegulatoryInformation/Guidances/ ucm088714.pdf (accessed Jan 12, 2012). 